\title{
Development of automatic production line for certain length iron wire
}

\author{
Xie Xu ${ }^{\mathrm{a}}$, Jianyang Zhu ${ }^{\mathrm{b}}$, Shuzhen Liu ${ }^{\mathrm{c}}$,
}

School of Mechanical Engineering, Ningbo University of Technology, Ningbo, China, 315016

a782404298@qq.com , b984259378@qq.com, cshuzhenl@163.com

Keywords: iron wire, automatic mechanism, cutting, length control

\begin{abstract}
A certain length of iron wire is widely applied in industry. In order to improve efficiency, an automatic production line, which includes transmitting, length control and cutting mechanism for iron wire, is developed by using Method of three dimensional design. The line is applied in one of processes of the production of PMMA in Ningbo. Practical application shows that this production line is stable and reliable, and is helpful to improve quality and quantity of production.
\end{abstract}

\section{Introduction}

Iron wire is available in market as bundles, as shown in fig.1. In the producing of poly methylmetharylate (PMMA), a certain length of iron wire, with the diameter of $0.6 \mathrm{~mm}-1.3 \mathrm{~mm}$, is needed to produce shape mold.

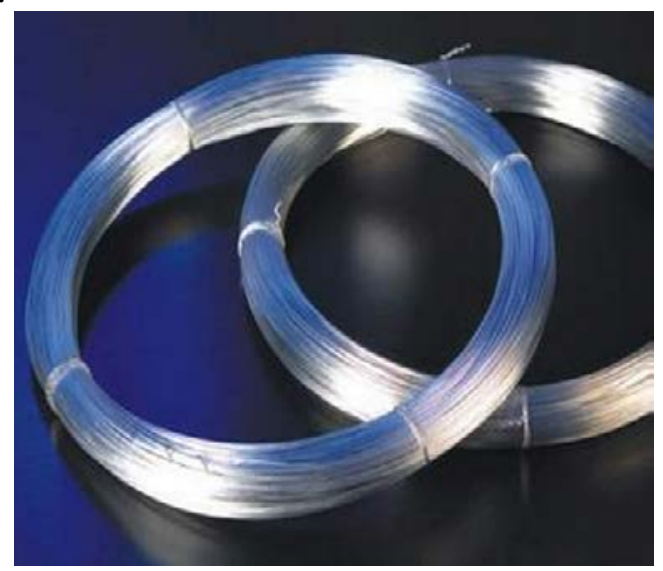

Fig.1 Iron wire

Yali etc. developed a cam swing-rod shearing mechanism [1]. Other kinds of iron wire cutting and measuring machines were designed by engineers and scholars [2-5]. Most of them are single function or structure complex. In this paper, an automatic transmitting, length control and cutting production line is developed. Firstly, the length of iron is set up on the length control worktable. Then, the iron wire is transmitted by a roller and the cutting machine moves to cut the wire at the right point.

\section{Design of one-way feed mechanism}

The working principle of the iron wire automatic feed mechanism is shown in Fig.2 and Fig.3. Components 2, 3, 4 are positive electrode 2, negative electrode 3 and negative electrode 4 respectively. The iron wire is passed through electrode 2 and 3, and the right side of it is put on the electrode 1 . The left side of the iron wire is twined on the roller, which is driven by a speed motor. 


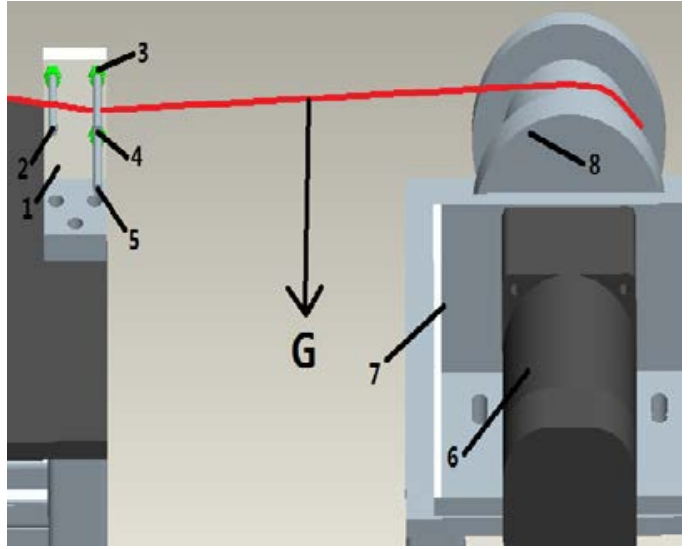

(a)

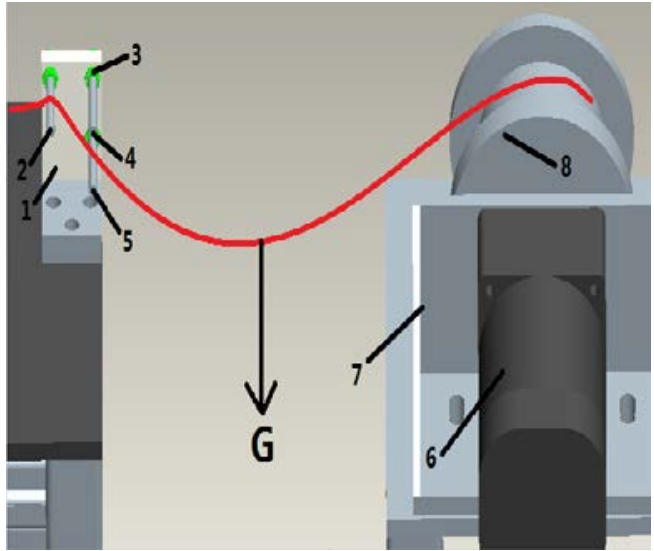

(b)

1. Holder for electrode (nylon) ; 2. No. 2 electrode; 3. Locknut;

4. No.3 electrode; 5. No.4 electrode; 6. Speed motor; 7. Board of motor; 8. Roller

Fig. 2 The state of wire when it is conveyed from roller

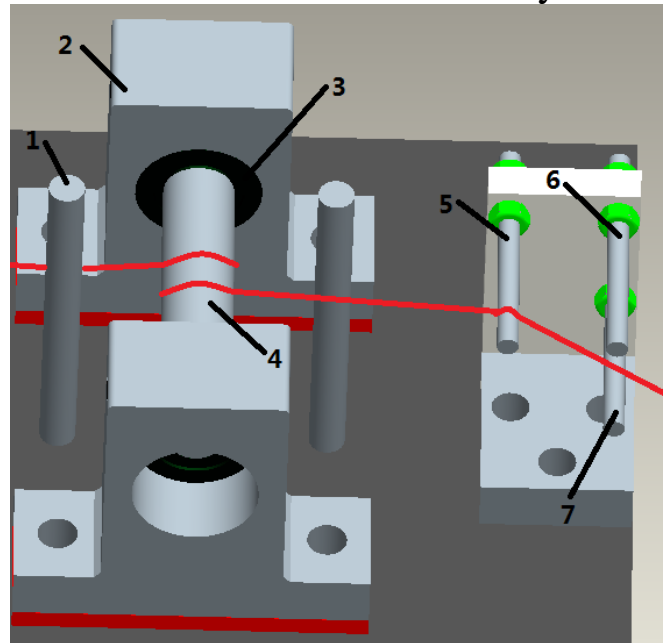

1. Position-limited pole; 2. Bearing seat; 3 One-way bearing;

4. One-way shaft; 5. No. 2 electrode; 6. No.3 electrode; 7. No.4 electrode

Fig. 3 The principle diagram of one-way feed mechanism

When the pneumatic clamp grabs the right end of the iron wire, it contacts the negative electrode and the iron wire becomes tight. At this moment, the electrode 2 and 3 are conducted by the iron wire between them and a signal is sent to the speed motor for starting to feed wire. For the one-way feeding, a mechanism, which contains position limited pole, one-way shaft and one-way bearing, is designed, as shown in Fig.3. The iron wire passes by the outside of the right position-limited pole and the inside of the left position-limited pole. At the same time, the wire is twined one circle on the one-way shaft. Thus, the iron wire could not be crossed over the one-way shaft or stuck on it. As the speed motor begins to transmit the wire, the weight of the iron wire between the electrode and the roller becomes high until the wire contacts with the electrode 4 . Under this condition, the electrode 2 and electrode 4 becomes a circuit loop and a signal is sent to motor for ceasing the transmission. After this, next operating cycle begins.

\section{Design of length control worktable}

The working principle of the length control system is shown in Fig.5. A reciprocal screw rod is used, on which there are two screw-nuts installed for fixing holding-plates. Tow ends of the holding-plates are installed on the linear guides, which moves straightly and reciprocally. The pneumatic clamp 13 is fixed on the left holding-plate and the wire holder 12 is fixed on the right one. When turning the hand-wheel, the screw drives the two screw-nuts and the holding-plates on it to move in opposite direction. Thus, the length of iron wire between the two holding-plates is changed. By using reciprocal screw rod, it is easy to keep the midpoint of the two screw-nuts in the fixed 
location and make the iron wire go to the next working position precisely. When the length of cutting wire is set up, the pneumatic manipulator pulls the iron wire to the set point as shown in fig.6.

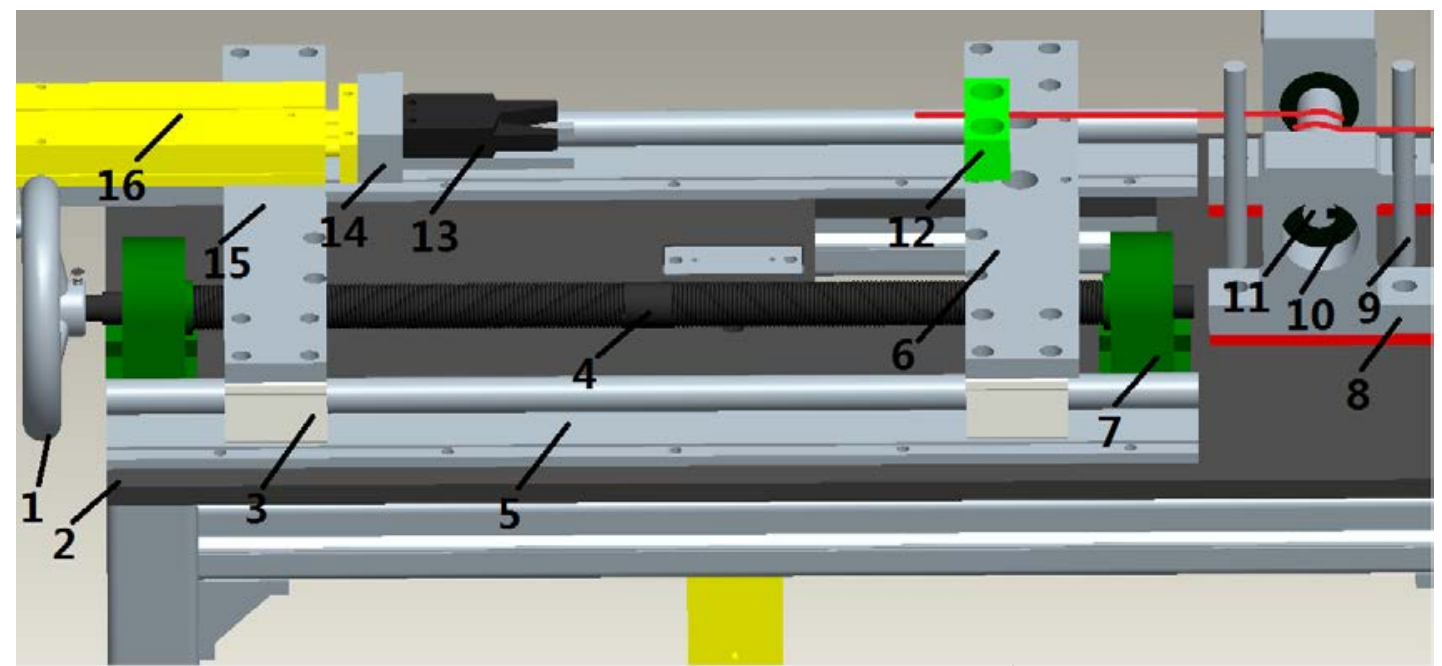

1. Hand-wheel; 2.Base plate; 3.Slide block; 4. Reciprocal screw rod; 5. Guideway; 6. Right holding-plate; 7. Bearing(CUPL202) ; 8.One-way bearing seat; 9. Position-limited pole; 10. One-way bearing; 11.One-way shaft; 12 . Iron square with a hole for wire going through; 13. Pneumatic clamp; 14. Base for fixing pneumatic clamp; 15. Left holding-plate; 16. Direct-acting cylinder

Fig.5 Iron wire length control system

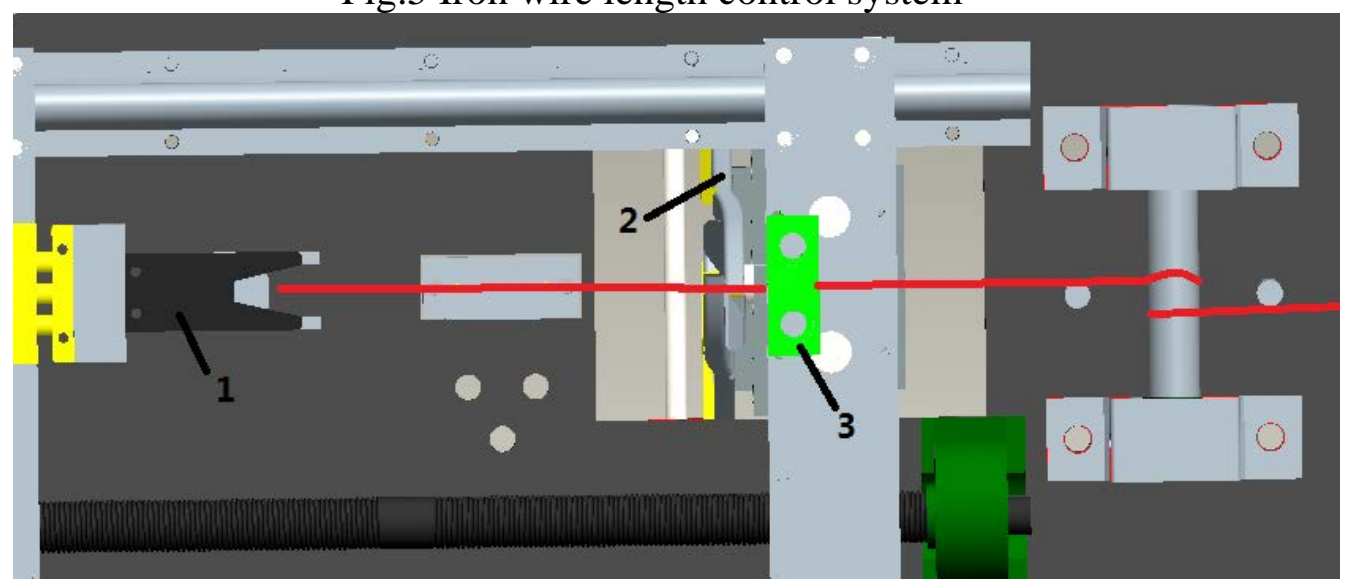

1. Pneumatic clamp; 2. Shearing mechanism; 3 Iron square with a hole for wire going through Fig.6 Diagram of pneumatic manipulator operation

\section{Design of shearing mechanism}

The principle of shearing mechanism is shown in Fig.7. The scissors are installed on the end of the moving cylinder 1 , which drives the scissors mechanism move up and down. When the setting length of wire is ready, the moving cylinder drives the scissors up to the cutting position. Then, the cutting cylinder drives the scissor to cut the wire. The scissor mechanism is a four-bar linkage.

\section{Conclusion}

In order to meet the requirements of PMMA production, an automatic production line for certain length iron wire is developed. One-way feeding mechanism is designed to transmit the iron wire from the roller. Pneumatic clamp is used to grab the wire and pull it. Length control mechanism can be used to set up three different length of wire. The reciprocal screw rod is used to keep the wire in the fixed position and is benefit for next processing. Scissors mechanism driven by pneumatic cylinder is designed. It is installed under the length control board so as to save room for the whole 
system. This automatic production line has been manufactured and applied in Ningbo. The production efficiency of PMMA has been improved by using it. It can also be used to other industry areas.
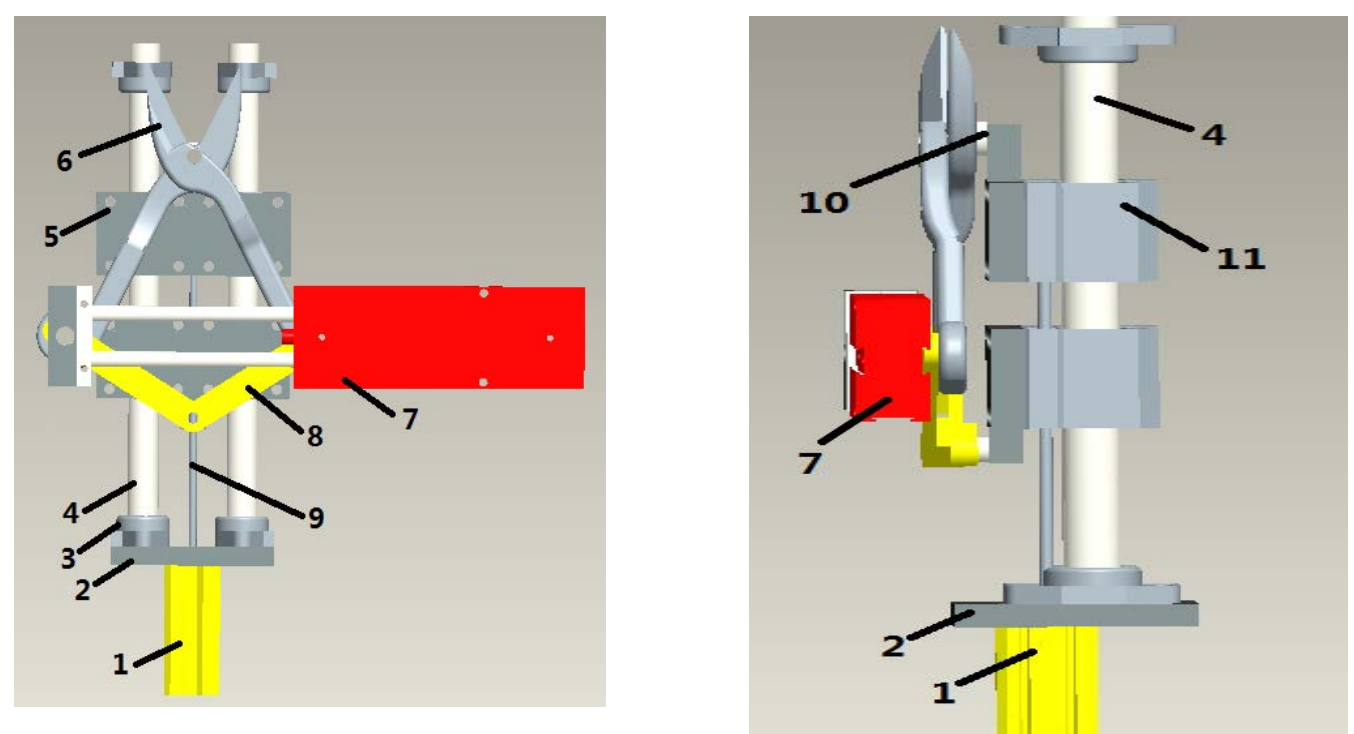

1. Pushing cylinder; 2. Base for fixing cylinder; 3. Base for shaft; 4. Shaft; 5. Sliding board; 6.Scissors; 7. Shearing cylinder; 8. Connecting rod; 9 pushing rod; 10. Gasket; 11. Slider

Fig.7 Working principle of automatic cutting mechanism

\section{Acknowledgments}

This work was supported by the Zhejiang Provincial Natural Science Foundation of China under Grant LQ13E050005.

\section{References}

[1]. Yali Yi, Herong Jin, Xiuchun Lu. Design and parameters calculation of cam swing rod shearing mechanism, Mechanical design and innovation, (2013) No.11, p.17-18.

[2]. Nu Qiao, Zhiming Wu. Design of mold for wire forming. Mold industry. Vol. 35 (2009) No.11, p. 36-39.

[3]. Lan Dong, Fuxiang Tian. Steel wire cutting and bending die. Mold industry. (2005) No.3, p. 28 29.

[4]. Jihui Zhong, Zuowen Wang, Xiaoping Meng. Optimal design of major components of the steel wire rope cutter. Forest engineering. (2005) No.6, p.13-14.

[5]. Hongxin Wang, Lin Li, Rongsheng Chang etc. Shearing mechanism design of two mutually twisted flat screw wires. Mechanical design. (2004) No.45, p.25-26.

[6]. Yan Guo, Jiatong Bao. Designed and Implementation of a Semiautonomous Search Robot.Proceedings of the 2009 IEEE International Conference on Mechatronics and Automation. 2009. p. 4621-4626.

[7]. GuoShing Huang, ChiouKou Tung, HsiungCheng Lin, and Shun-Hui Hsiao. Inverse Kinematics Analysis Trajectory Planning for a Robot Arm. Proceedings of 2011 8th Asian Control Conference (ASCC), 2011. p. 965- 970. 